\title{
Influence de la recombinaison sur la variabilité génétique. Il. Étude par simulations
}

\author{
J Kervella ${ }^{1}$, N Robert 2*, G Fouilloux ${ }^{3}$ \\ 1 INRA, station de recherches fruitières méditerranéennes, BP 91, F84143 Montfavet Cedex; \\ 2 INRA, station d'amélioration des plantes, domaine de Crouelle, F63039 Clermont-Ferrand Cedex; \\ ${ }^{3}$ INRA, station d'amélioration des plantes, route de Saint-Cyr, F78000 Versailles, France
}

(Reçu le 22 février 1991; accepté le 6 janvier 1993)

\begin{abstract}
Résumé - Pour compléter une étude expérimentale portant sur l'intérêt, pour l'amélioration des plantes, d'un gène élévateur des fréquences de recombinaison, deux études par simulation ont été réalisées. Dans la première étude, des descendances de 10 individus ont été dérivées par une génération d'autofécondation ou par un plan circulaire de croisement, à partir de quatre populations, dans des cas de fréquences moyennes de recombinaison élevées, intermédiaires et faibles. Les distributions des variances génétiques des descendances issues d'une même population ont été comparées. Dans les deux types de descendances, les distributions des variances génétiques se recouvrent largement, que la population présente un excès d'associations en couplage ou en répulsion. II risque donc d'être difficile de mettre expérimentalement en évidence l'effet de différences de fréquences de recombinaison sur la variabilité génétique de caractères quantitatifs. Dans la seconde étude, un programme de sélection récurrente a été simulé pour des fréquences moyennes de recombinaison élevées, intermédiaires, et faibles. Pour la fréquence de recombinaison la plus faible, le progrès génétique est plus lent, légèrement moins important, et la variance génétique plus élevée. Par contre, de même que pour la simulation précédente, les fréquences de recombinaison élevées et intermédiaires donnent des résultats similaires du point de vue du progrès génétique et de la variance génétique. Un gène élevant les taux de recombinaison n'est donc intéressant que si le linkage est très étroit ou les croisements difficiles. Sinon, un plan d'intercroisement assure une gestion efficace de la variabilité.
\end{abstract}

simulation / recombinaison / variabilité génétique / variance / couplage / répulsion / sélection récurrente

Summary - Influence of recombination on genetic variability: II. Simulation studies. Two stimulation studies were carried out to complement an experimental study of the possible interest for plant breeders of an allele increasing recombination rates. In the first simulation study, 4 populations of 10 individuals were simulated in the case of high (45\%), medium (19.6\%) and low (6.9\%) recombination frequencies. Ten-plant populations were derived by one generation, either from selfing or from crossing in a circular mating design, from each of the 4 populations. For each initial population, the distribution of the genetic variances in the populations obtained by one mating system overlapped widely in the 3 cases of recombination rates considered, whether the initial population presented an excess of coupling phase, or an excess of repulsion phase. An experimental assessment of the effect of recombination rate differences on genetic variability of quantitative traits is therefore likely to be difficult. The second study simulated a recurrent selection program in the case of high (45\%), medium (19.6\%) and low (9\%) recombination rates. The mean genetic value increased somewhat more slowly and reached a slightly lower value for low recombination rates, whereas the genetic variance remained the highest after the second selection cycle. The evolutions in genetic mean values and genetic variances were almost identical for medium and high recombination frequencies. Thus, an allele which increases recombination rates will only be of interest in the case of close linkage or difficult crossing. Otherwise, a circular mating design manages efficiently the initial germplasm.

simulation studies / recombination / genetic variability / variance / coupling phase / repulsion phase / recurrent selection program

\footnotetext{
* Correspondance et tirés à part
} 


\section{INTRODUCTION}

Les travaux de Mather $(1941,1942)$ et de Spiess et Allen (1961) laissaient espérer une augmentation de la variance génétique lorsque la recombinaison est favorisée. Cependant, une étude expérimentale chez le pétunia des effets de l'allèle $R m 1$, élévateur des fréquences de recombinaison, n'a pas permis de révéler une influence de cet allèle sur la variance génétique de 5 caractères quantitatifs (Robert et al, 1993).

Pour interpréter nos résultats expérimentaux sur pétunia et les compléter, 2 études par simulations ont été effectuées :

- la première est destinée à simuler nos expérimentations ; elle concerne l'influence du taux de recombinaison sur la variance génétique de populations d'effectif faible, à l'issue d'une génération de croisement selon un plan circulaire, ou d'une génération d'autofécondation. Dans cette simulation, la répartition des allèles le long des chromosomes, peu maîtrisable expérimentalement, a été prise en considération. En effet, divers travaux théoriques ont montré que les conséquences de la recombinaison diffèrent selon l'importance du couplage et de la répulsion (Miller et Rawlings, 1967; Mather et Jinks, 1971; Gallais, 1974; Snape, 1976; Snape et Simpson, 1981). De plus, pour le sélectionneur, l'impact de la recombinaison dépend de l'organisation de la variabilité qu'il manipule : si la recombinaison brise les liaisons entre allèles favorables et défavorables et favorise ainsi les transgressions, elle détruit aussi les associations d'allèles favorables;

- la seconde simulation vise à apprécier l'effet du taux de recombinaison à plus long terme, dans le cadre d'un programme de sélection récurrente que nous ne pouvions réaliser sur notre matériel végétal.

\section{MATÉRIEL ET MÉTHODES}

Le programme de simulations utilisé est GENAMEL1, écrit par G Fouilloux. Les populations créées par ce programme sont constituées d'individus portant 40 loci qui contrôlent un caractère unique. La taille des populations peut être choisie, les effets (additivité, dominance, épistasie) de chaque allèle, l'héritabilité au sens large du caractère peuvent être paramétrés. À partir de ces paramètres, une valeur génétique et une valeur phénotypique sont attribuées à chaque individu.

\section{Taux de recombinaison}

Dans nos simulations, les 40 loci ont été répartis sur 5 chromosomes portant 8 loci chacun. Dans chaque étude, 3 cas, correspondant à des fréquences de recombinaison intra-chromosomiques fortes, intermédiaires et faibles, ont été considérés. Dans la suite de l'article nous ferons référence à ces cas par l'intermédiaire des moyennes arithmétiques des taux de recombinaison inter- et intra-chromosome. Dans la première étude, les 3 cas correspondent respectivement à des taux de recombinaison entre loci successifs le long d'un chromosome de 50\%, 50\%, 50\%, 50\%, $10 \%, 50 \%$, 50\% (moyenne 45\%); 40\%, 3\%, 20\%, $10 \%, 0.5 \%, 4 \%, 30 \%$ (moyenne $19,6 \%$ ) et $2 \%, 0,15 \%$, $1 \%, 0,5 \%, 0.02 \%, 0.2 \%, 1,5 \%$ (moyenne $6,9 \%$ ). Dans la seconde étude, des taux de recombinaisons de $8 \%$, $0,6 \%, 4 \%, 2 \%, 0,1 \%, 0,8 \%, 6 \%$ (moyenne $9 \%$ ) ont été préférés dans le dernier cas, pour que des recombinaisons se produisent effectivement.

\section{Déterminisme génétique}

Quatre formes alléliques, correspondant à 4 valeurs d'effets additifs, peuvent exister à chaque locus. Les 2 formes correspondant aux valeurs additives élevées sont dites favorables, les 2 autres défavorables. Pour $40 \%$ des loci, seuls les effets additifs interviennent. Pour les autres loci, la valeur génotypique d'un individu est en outre déterminée par des interactions alléliques intra-locus (effets de dominance partielle $(10 \%$ des loci) ou totale ( $50 \%$ des loci) de l'allèle favorable), et, dans le cas de la seconde étude, s'ajoutent des interactions entre loci (épistasie de type additive $x$ additive, dominance $x$ additive et dominance $x$ dominance).

Dans la second étude, l'héritabilité au sens large a été prise égale à 1 , une héritabilité plus faible ne modifiant pas les conclusions.

\section{Déroulement des simulations}

\section{Influence du taux moyen de recombinaison sur la variance génétique de populations de taille réduite}

Dans cette première étude, les populations sont constituées de 10 individus. Les conséquences de la recombinaison étant a priori différentes selon l'importance du couplage et de la répulsion, nous avons travaillé avec 4 populations mères, décrites au tableau I, différant par la répartition des allèles le long des chromosomes. Ainsi, le rapport du nombre de loci consécutifs portant des allèles ayant même effet additif sur le caractère, au nombre total de loci, varie de 0 à $94,2 \%$. Les populations $P_{1}$ et $P_{2}$ présentent un excès d'association en couplage, les populations $P_{3}$ et $\mathrm{P}_{4}$ un excès d'association en répulsion. 
Tableau I. Description des populations initiales de l'étude sans sélection : moyenne (MV) et écart type $(\sigma V)$ de la variance génétique, valeurs minimales et maximales (le taux de couplage est le pourcentage de loci consécutifs ayant même effet sur le caractère).

\begin{tabular}{lcccr}
\hline & $P_{1}$ & $P_{2}$ & $P_{3}$ & \multicolumn{1}{c}{$P_{4}$} \\
\hline Taux couplage & $94,2 \%$ & $57,7 \%$ & $16,6 \%$ & $0 \%$ \\
Moyenne & 354,0 & 349,9 & 376,9 & 367,4 \\
Variance & 1364,4 & 802,5 & 1475,0 & 1780,7 \\
\hline
\end{tabular}

Chaque simulation a consisté, à partir d'une des populations mères, à obtenir une population fille de 10 individus, par SSD ou par un plan circulaire de croisements PCC (protocole schématisé sur la figure 1) et à en calculer la variance génétique. Pour chaque triplet (population mère, fréquence de recombinaison, type de descendance), 150 simulations ont été réalisées afin de connaître la dispersion des variances génétiques et d'obtenir une estimation fiable de leur moyenne.

\section{Influence du taux moyen de recombinaison sur le progrès génétique obtenu par sélection récurrente}

Dans cette seconde étude, chaque simulation correspond à un programme de sélection récurrente mené pendant 15 cycles pour les fréquences de recombinaison élevées et intermédiaires, pendant 30 cycles pour les fréquences faibles.

La population initiale est constituée de 144 individus tirés au hasard d'une population panmictique de référence, sans déséquilibre de liaison. Tous les cycles se déroulent de façon identique (fig 2). Une sélection phénotypique individu-famille est pratiquée sur les 144 familles de 10 individus obtenues par autofécondation des individus de départ: le meilleur individu de cha-

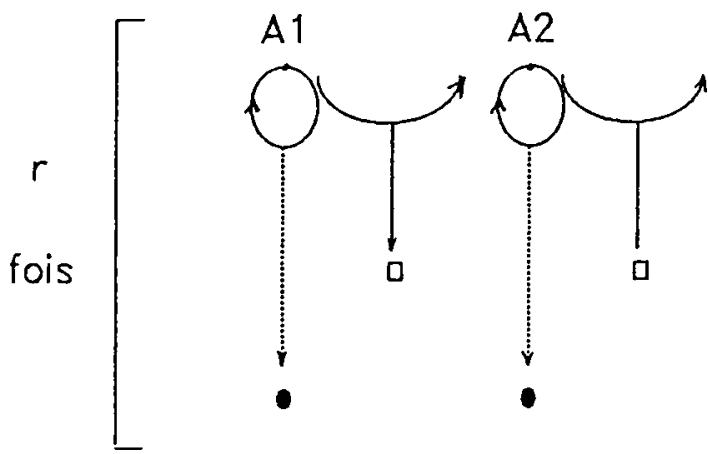

cune des 24 meilleures familles est retenu. Afin de simuler le cas le plus général, où la multiplication végétative n'est pas possible, ce sont les descendants en autofécondation (un par individu sélectionné) qui sont à la base du cycle suivant. Ces descendants sont croisés selon un plan conique à 3 étages. Les taux de multiplication étant de 3 pour le deuxième étage et de 2 pour le dernier, 144 individus $S_{0}$ sont obtenus à l'issue de ces intercroisements et composent la population de départ du cycle suivant. La moyenne et la variance des populations au départ de chaque cycle sont calculées.

Quinze simulations, démarrant chacune d'une population panmictique différente, ont été réalisées. Les données présentées pour chaque cycle de sélection sont les moyennes des 15 simulations.

\section{RÉSULTATS ET DISCUSSION}

\section{Étude sans sélection}

Les distributions des variances génétiques des 150 populations obtenues pour chacune des populations de départ par autofécondation ou par plan circulaire de croisement ont été représentées par la technique du boxplot (la boîte est définie par les premier et troisième quartiles de la distribution) (fig 3). Les moyennes, écarts-types, minima et maxima de ces distributions sont récapitulés au tableau II.

Les taux de recombinaison influencent de la même façon les variances génétiques des 2 types de descendances. Pour une population de départ et un type de descendance donnés, les variances génétiques des populations obtenues pour chacun des taux de recombinaison sont très variables et leurs distributions se recouvrent

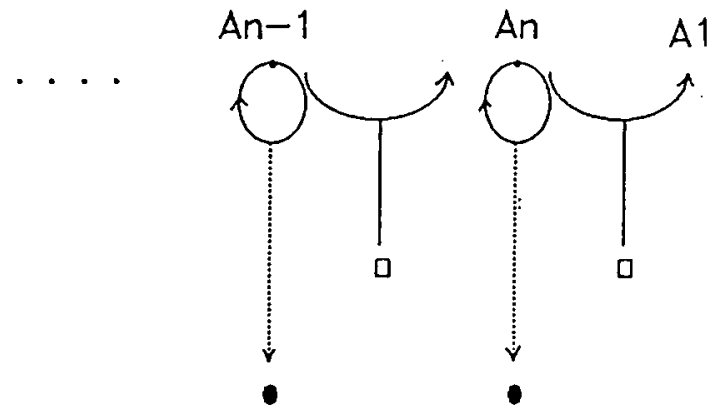

Individu de la population PCC $\square$

individu de la population SSO

Fig 1. Schéma d'obtention de 150 populations, à partir des populations conduites sans sélection $P_{1}, P_{2}, P_{3}$ et $P_{4}$. 


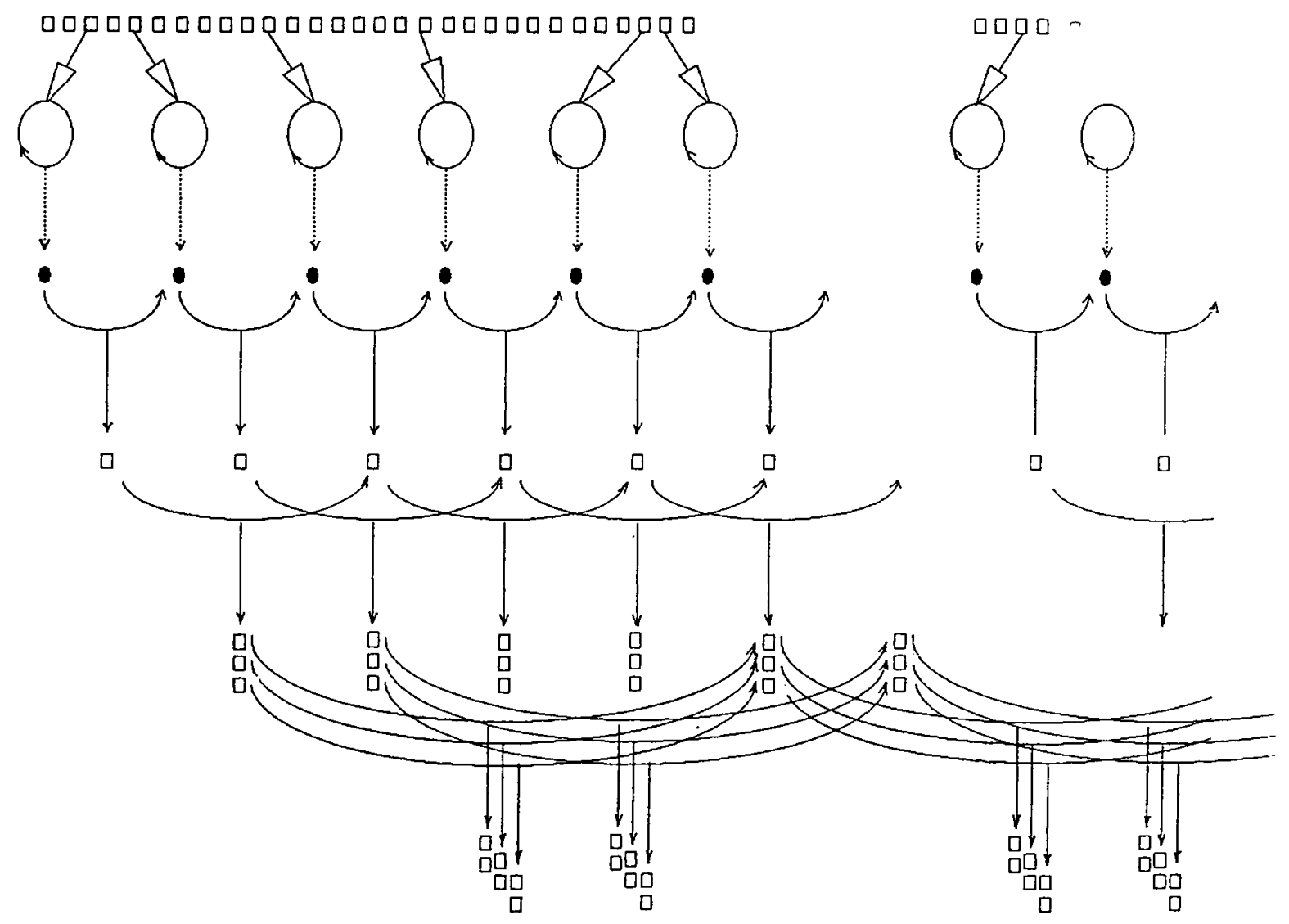

Fig 2. Description d'un cycle de sélection récurrente simulé.

Tableau II. Moyenne, écart type, minimum et maximum de la variance génétique pour les populations en couplage $\left(P_{1}\right.$ et $\left.P_{2}\right)$ et en répulsion $\left(P_{3}\right.$ et $\left.P_{4}\right)$.

\begin{tabular}{|c|c|c|c|c|c|c|c|c|c|}
\hline & & \multicolumn{2}{|c|}{$P_{1}$} & \multicolumn{2}{|c|}{$P_{2}$} & \multicolumn{2}{|c|}{$P_{3}$} & \multicolumn{2}{|c|}{$P_{4}$} \\
\hline & & $S S D$ & $P C C$ & SSD & $P C C$ & $S S D$ & $P C C$ & SSD & $P C C$ \\
\hline \multirow[t]{4}{*}{$6,9 \%$} & MV & $3005,8^{a}$ & $2379,6^{a}$ & $1351,2^{a}$ & $1154,9^{a}$ & $1323,0^{a}$ & $796,5^{a}$ & $1044,7^{a}$ & $743,9^{a}$ \\
\hline & $s V$ & 1655,6 & 1138,3 & 733,5 & 516,7 & 493,4 & 313,8 & 369,5 & 330,4 \\
\hline & $\operatorname{mini}$ & 717,9 & 526,4 & 155,7 & 218,3 & 422,7 & 127,6 & 270,2 & 146,9 \\
\hline & $\operatorname{maxi}$ & 10390,0 & 6225,8 & 3830,0 & 3202,9 & 2731,1 & 1794,9 & 2188,2 & 1782,7 \\
\hline \multirow[t]{4}{*}{$19,6 \%$} & MV & $1917,0^{b}$ & $1669,2^{b}$ & $1016,1^{b}$ & $829,0^{b}$ & $1007,1^{b}$ & $683,1^{b}$ & $883,4^{b}$ & $619,4^{\mathrm{b}}$ \\
\hline & sV & 932,6 & 794,9 & 461,0 & 356,1 & 361,4 & 279,4 & 279,6 & 259,4 \\
\hline & $\operatorname{mini}$ & 447,2 & 258,0 & 200,9 & 144,4 & 414,0 & 157,5 & 324,7 & 124,5 \\
\hline & $\operatorname{maxi}$ & 5244,7 & 4905,2 & 2544,5 & 2141,8 & 2442,6 & 1636,2 & 1808,8 & 1424,0 \\
\hline \multirow[t]{4}{*}{$45 \%$} & MV & $1261,3^{c}$ & $862,5^{c}$ & $786,7^{c}$ & $638,1^{c}$ & $1082,3^{b}$ & $721,4^{b}$ & $949,9 \mathrm{~b}$ & $668,8^{\mathrm{b}}$ \\
\hline & sV & 537,1 & 432,0 & 288,8 & 286,3 & 365,0 & 316,0 & 312,3 & 277,1 \\
\hline & $\operatorname{mini}$ & 263,6 & 173,8 & 209,2 & 118,0 & 420,2 & 150,1 & 356,0 & 125,1 \\
\hline & $\operatorname{maxi}$ & 3118,0 & 2605,8 & 1488,9 & 1666,7 & 2461,9 & 2057,7 & 1912,4 & 1780,7 \\
\hline
\end{tabular}

$M V$ : moyenne; $\sigma V$ : écart type; mini : variance minimale; maxi : variance maximale; les moyennes affectées de lettres différentes diffèrent statistiquement, les comparaisons ont été faites 2 à 2 par un test de $t$ ou $t^{\prime}$. 

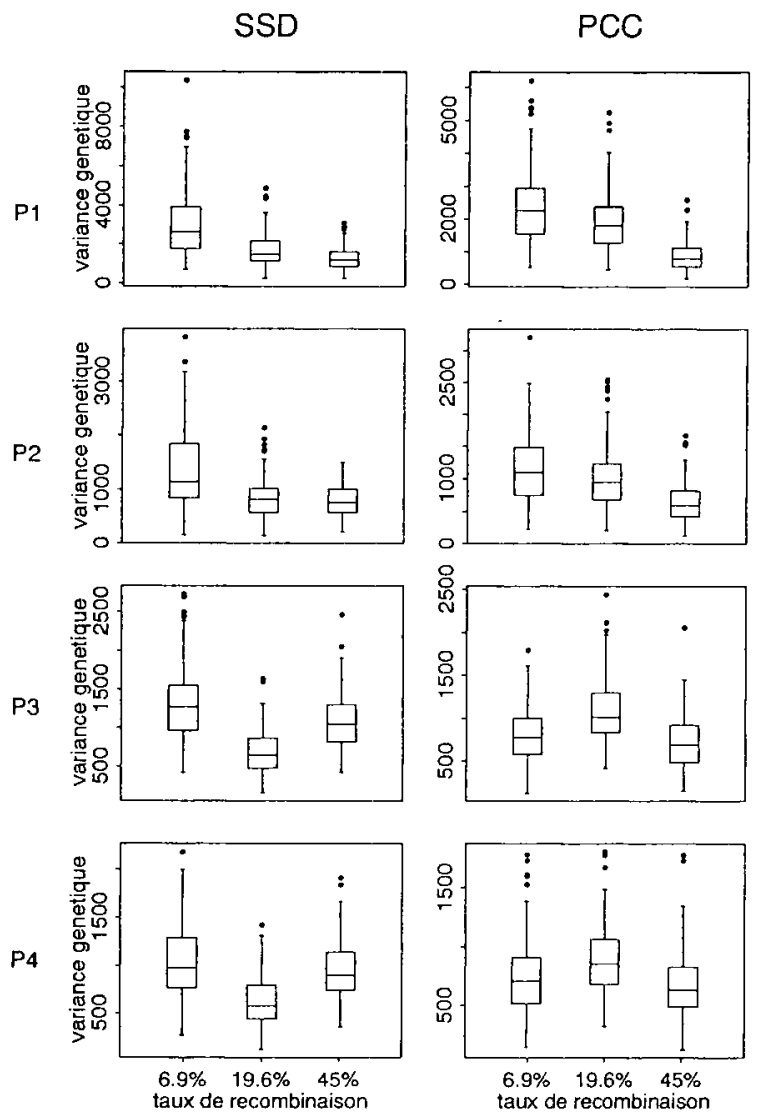

Fig 3. Distribution des variances génétiques des populations obtenues après une génération de SSD ou un plan circulaire de croisement ( $P C C$ ) pour les populations $P_{1}, P_{2}, P_{3}$ et $P_{4}$ et des fréquences de recombinaison élevées $(45 \%)$, intermédiaires $(19,6 \%)$ et faibles $(6,9 \%)$.

largement. Pour les populations $P_{1}$ et $P_{2}$, présentant un excès de couplage, elles sont d'autant plus variables que le taux de recombinaison est faible. Par contre, pour les populations en répulsion $P_{3}$ et $P_{4}$, la variabilité ne semble pas dépendre du taux de recombinaison.

Les tests de comparaison de moyennes (tests $t$ ou t) montrent que, malgré cette variabilité, la variance génétique moyenne pour le taux de recombinaison $6,9 \%$ est dans tous les cas significativement supérieure à celles obtenues pour les taux de recombinaison plus élevés mais ceci est plus marqué pour les populations présentant un excès de couplage (populations $P_{1}$ et $P_{2}$ ) que pour les populations présentant un excès de répulsion.

Pour un type de descendance et une population de départ donnés, la variance génétique moyenne obtenue pour le taux de recombinaison $19,6 \%$ est significativement supérieure à celle obtenue pour le taux de recombinaison $45 \%$ dans le cas des populations en excès de couplage. Pour les populations en répulsion, les va- riances génétiques pour ces 2 taux de recombinaison ne diffèrent pas significativement, quel que soit le type de descendance. Cependant, la valeur de la variance génétique moyenne est à chaque fois plus élevée pour le taux de recombinaison $45 \%$.

\section{Influence du taux de recombinaison sur le progrès génétique en sélection récurrente}

\section{Évolution du progrès génétique avec les 3 taux de recombinaison pour 15 cycles de sélection (fig 4)}

Quel que soit le taux de recombinaison, on constate une élévation de la moyenne de la population. L'évolution de la moyenne est identique pour les taux $19,6 \%$ et $45 \%$ : l'augmentation, rapide dans les 4 premiers cycles, se ralentit ensuite et la moyenne se stabilise vers le $11^{\mathrm{e}}$ cycle. Pour le taux $9 \%$, l'augmentation de la moyenne est plus faible dans les premiers cycles. Par contre, elle se poursuit jusqu'au $23^{3}$ cycle environ (données non présentées). II en résulte que l'écart entre les moyennes des populations pour les taux $19,6 \%$ et $45 \%$ d'une part, $9 \%$ d'autre part, se creuse durant les 7 premiers cycles de sélection, pour s'amenuiser ensuite. Au $23^{\mathrm{e}}$ cycle, les valeurs génétiques moyennes des populations sont respectivement 636,635 et 620 pour les taux de recombinaison $45 \%, 19,6 \%$ et $9 \%$.

\section{Évolution des variances génétiques pour 15 cycles de sélection (fig 5)}

Les variances génétiques sont du même ordre de grandeur au départ pour les 3 taux de recom-

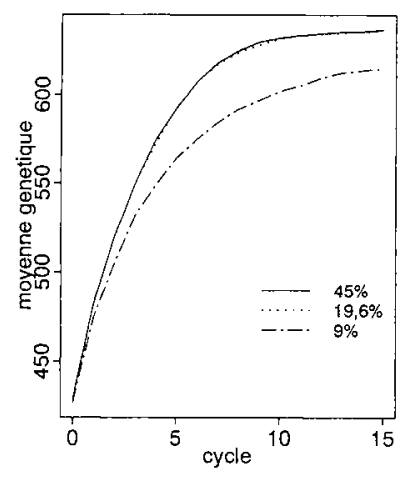

Fig 4. Évolution de la valeur génétique moyenne des populations en sélection récurrente pour des fréquences de recombinaison élevées $(45 \%)$, intermédiaires $(19,6 \%)$ et faibles $(9 \%)$ (les résultats présentés sont les moyennes de 15 répétitions). 


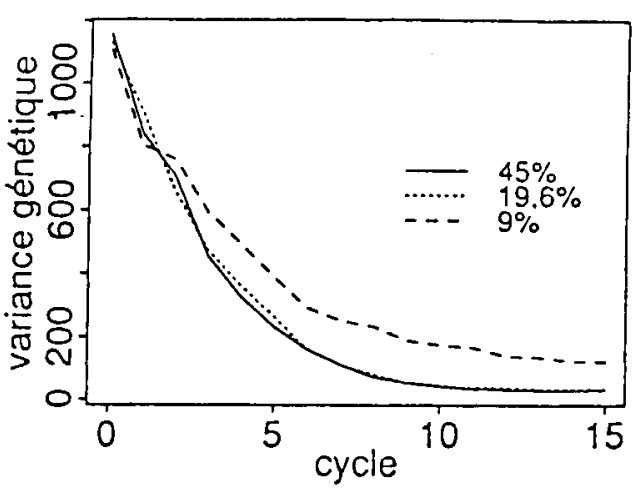

Fig 5. Évolution de la variance génétique moyenne des populations en sélection récurrente pour des fréquences de recombinaison élevées $(45 \%)$, intermédiaires $(19,6 \%)$ et faibles $(9 \%)$ (les résultats présentés sont les moyennes de 15 répétitions).

binaison. Elles varient de manière identique pour les taux $19,6 \%$ et $45 \%$. Elles diminuent fortement pendant les 3 premiers cycles, puis de plus en plus lentement pour se stabiliser aux alentours de 30 vers le $11^{\mathrm{e}}$ cycle, au moment où le progrès génétique cesse.

Après le premier cycle de sélection, la variance génétique est plus élevée pour le taux de recombinaison $9 \%$ que pour les 2 autres taux et elle diminue plus lentement. Elle se stabilise également plus lentement et à un niveau plus élevé (aux alentours de 60).

Pour ce taux de $9 \%$, la décroissance de la variance pour une population donnée présente des irrégularités qui n'apparaissent plus quand on considère la variance moyenne sur l'ensemble des populations. Comme pour la valeur moyenne, l'écart entre les populations pour les taux $19,6 \%$ et $45 \%$ d'une part, $9 \%$ d'autre part, est maximal vers le $8^{\mathrm{e}}$ cycle de sélection.

\section{DISCUSSION}

\section{Interprétation génétique}

Dans cette interprétation, nous nous référerons fréquemment aux travaux de Mather et Jinks (1971). Ces auteurs ont en effet développé une analyse génétique à partir d'un modèle faisant intervenir des effets additifs, de dominance et d'épistasie, tels qu'ils sont définis dans le programme de simulation.

\section{Influence du taux de recombinaison sur la variance génétique en l'absence de sélection}

Nos résultats sont en accord avec les calculs effectués par Mather et Jinks (1971) concernant l'influence du taux de recombinaison sur la variance génétique en cas de déséquilibre de liaison. Ces calculs, effectués pour une population $F_{2}$, montrent que, par rapport à l'équilibre de liaison, les composantes de la variance faisant intervenir des effets additifs sont augmentées pour les associations en couplage et diminuées pour les associations en répulsion. De plus, en valeur absolue, l'écart de chaque composante de la variance par rapport à la valeur à l'équilibre est d'autant plus grand que le taux de recombinaison est faible et que l'excès de couplage ou de répulsion est important. En conséquence, en présence de couplage, la variance additive est toujours supérieure à la valeur à l'équilibre et diminue lorsque la recombinaison augmente ; par contre en présence de répulsion, cette variance, inférieure à la valeur à l'équilibre, augmente lorsque la recombinaison augmente.

Pour les composantes faisant intervenir des effets de dominance, le signe de l'écart à l'équilibre ne dépend pas du type d'associations : il est positif ou négatif pour une paire de loci donnée suivant que les effets de dominance à ces loci sur le caractère considéré sont de même signe ou de signes opposés. Pour une population quelconque, Gallais (1974) aboutit aux mêmes conclusions par une autre méthode de calcul. Lorsque les effets de dominance sont tous de même signe, une élévation des taux de recombinaison conduit toujours à une baisse de la variance génétique en présence de couplage. Par contre, l'évolution de cette variance est plus difficilement prévisible en cas de répulsion, car elle dépend de l'importance des effets de dominance par rapport aux effets additifs.

Selon le modèle de Mather et Jinks, une augmentation du taux de recombinaison, dans le cas de populations initiales présentant un excès d'associations en répulsion, n'entraîne une augmentation de la variance génétique qu'en l'absence d'effets de dominance, cas le plus fréquemment considéré (Miller et Rawlings, 1967; Snape, 1976; Snape et Simpson, 1981).

Dans nos simulations, les effets de dominance des différents loci sont de même signe. Les résultats sont en accord avec ceux de Mather et Jinks. En effet, la variance génétique moyenne 
des populations présentant un excès de couplage diminue lorsque les taux de recombinaison augmentent. De même, les écarts entre les moyennes des 3 taux de recombinaison sont plus prononcés lorsque l'excès de couplage est plus important.

Les résultats sur les populations présentant un excès de répulsion confirment la difficulté à prédire l'évolution de la variance génétique pour une telle situation. Selon l'importance relative des variations des composantes additive et de dominance, leur résultante, la variance génétique, peut en particulier diminuer puis augmenter légèrement lorsque le taux de recombinaison augmente, comme nous l'avons observé pour les populations $P_{3}$ et $P_{4}$.

Les résultats de Spiess et Allen (1961) ne semblent pas en accord avec l'approche théorique et nos simulations. Travaillant sur la viabilité chez la drosophile, ces auteurs observent une variance génétique plus forte lorsque la recombinaison a lieu. Un tel résultat serait attendu dans le cas d'additivité prédominante, en situation de répulsion. Les informations données par les auteurs ne confirment pas cette prédominance des effets d'additivité. Mais ils les évaluent au niveau chromosomique et non au niveau de chaque locus. De plus, les effets significatifs d'interaction entre chromosomes laissent supposer des effets d'épistasie importants, effets non pris en compte dans nos simulations. Cela pose le problème de l'adéquatión des modèles théoriques à la réalité biologique.

\section{L'influence du taux de recombinaison sur le comportement d'une population en sélection récurrente}

La réponse à long terme à la sélection est limitée par le déséquilibre de liaison induit par la sélection. Dans une population de taille réduite, les effets de consanguinité et de dérive résultant du faible effectif limitent également cette réponse (Verrier et al, 1990). En cas de sélection directionnelle, le déséquilibre de liaison induit consiste en un excès d'associations en répulsion. II est la cause essentielle de la diminution de la variance génétique additive dans les premiers cycles de sélection lorsque les effets de chacun des gènes sont faibles (Bulmer, 1971). Chaque étape de sélection tend à accroître ce déséquilibre mais, du fait des recombinaisons, chaque intercroisement tend à rétablir l'équilibre de liaison. Au bout de quelques générations, sous l'influence de ces 2 effets antagonistes, la variance génétique utilisable par la sélection atteint une valeur limite, d'autant plus faible que les taux de recombinaisons sont faibles (Bulmer, 1971).

Ce phénomène explique bien l'évolution du progrès génétique au cours des premières générations de sélection, et en particulier sa diminution rapide puis plus lente. II explique également le progrès plus faible et plus lent observé pour le taux de recombinaison de $9 \%$. II peut y avoir 2 raisons à l'absence de différence entre les taux de recombinaison de $45 \%$ et $19,6 \%$. D'une part, les conséquences d'une différence de taux de recombinaison sur la variance génétique d'équilibre sont de moins en moins sensibles quand le taux de recombinaison augmente (Bulmer, 1974). D'autre part, les 3 générations d'intercroisement sans sélection tendent à réduire les différences entre taux de recombinaisons par rapport à un cycle avec une seule génération d'intercroisement.

L'excès d'associations en répulsion conduit à une augmentation, décrite précédemment, de la composante de la variance faisant intervenir les effets alléliques de dominance, lorsque les taux de recombinaison décroissent et que les effets de dominance sont de même signe. Ceci peut expliquer la plus forte variance génétique observée pour le taux de recombinaison le plus faible. Notons que ce résultat est contraire aux conclusions de Bulmer (1971). D'après celles-ci, établies pour un nombre infini de loci et en l'absence d'épistasie, on attendrait aussi une diminution de la variance génétique totale lorsque le taux de recombinaison diminue, puisque l'évolution de la variance génétique totale serait identique à celle de la variance génétique additive.

Les effets de consanguinité résultant d'une taille réduite des populations conduisent à une fixation progressive des allèles aux différents loci. À long terme, ils contribuent donc à diminuer toutes les composantes de la variance génétique. Ces effets se cumulent de génération en génération et sont d'autant plus marqués que la sélection est efficace (Verrier et al, 1990).

II est donc probable que les effets d'une taille réduite des populations varient en fonction du taux de recombinaison. Ainsi, dans le cas du faible taux de recombinaison, où le progrès génétique est plus lent, ces effets se font sentir sur un plus grand nombre de générations. II est possible aussi que leurs conséquences en terme de fixation d'allèles défavorables soient plus impor- 
tantes : lorsque la recombinaison est peu fréquente, la fixation se fait par bloc chromosomique. Ces conséquences plus importantes de la taille réduite des populations dans le cas du faible taux de recombinaison pourraient expliquer la plus faible valeur génétique atteinte par la population lorsque le progrès génétique cesse.

Le fait que la variance génétique se stabilise à une valeur non nulle dans les derniers cycles montre toutefois que la fixation complète n'a pas été obtenue et suggère que l'effet de la dérive n'est pas prépondérant dans les différences observées entre taux de recombinaison. Ceci serait cohérent avec les faibles différences, entre taux de recombinaison, pour la valeur génétique moyenne atteinte lorsque la sélection cesse d'être efficace. Cet effet peu important de la dérive pourrait résulter des modalités de sélection et d'intercroisement adoptées, qui limitent les croisements entre apparentés.

La stabilisation de la variance génétique à une valeur non nulle, alors que le progrès génétique cesse, provient vraisemblablement de l'existence d'une composante épistatique dans la variance génétique : aux loci encore en ségrégation, le caractère plus ou moins favorable de chacun des allèles dépend plus du contexte génétique que de l'allèle lui-même, si bien que la sélection ne parvient plus à privilégier l'un des allèles.

\section{Conséquences pour l'étude expérimentale de la recombinaison}

Les simulations portant sur des populations d'effectifs faibles ont été réalisées pour être un appui à l'interprétation de l'étude expérimentale sur pétunia. Dans celle-ci, aucune différence significative n'apparaissait entre les variances inter-familles de groupes de plantes supposés différer par leurs taux de recombinaison, que les plantes soient conduites en autofécondation ou en croisement. Les résultats des simulations montrent qu'il n'est pas nécessaire de faire I'hypothèse d'un déterminisme des caractères mal adapté à l'étude entreprise (insensibilité à l'action de l'allèle $\mathrm{Rm}_{1}$ des segments chromosomiques concernés ou indépendance des loci) pour expliquer cette absence de différence. En effet, le large recouvrement des distributions des variances génétiques pour des taux de recombinaison moyens variant de $45 \%$ à $6,9 \%$ suggère que des effets d'échantillonnage peuvent être à l'origine de nos résultats. Cela d'autant plus que les effectifs des populations expérimentales sont plus faibles. Cela implique que pour mettre en évidence de façon expérimentale les effets d'un changement du taux de recombinaison sur la variabilité de caractères quantitatifs, un grand nombre de répétitions de la même expérience est nécessaire, ce qui risque d'être lourd.

Les résultats obtenus dans les 2 types de simulation (sans sélection et avec sélection récurrente) montrent que les variances génétiques des populations obtenues ne diffèrent que très peu pour des taux de recombinaison moyens de $45 \%$ et $19,6 \%$. En conséquence, même si l'allèle $\mathrm{Rm}_{1}$ augmente effectivement les taux de recombinaison entre les loci impliqués dans le déterminisme du caractère étudié, et modifie donc les combinaisons de gènes induites par la recombinaison, cela peut ne pas se traduire par des différences de la variance génétique. Une autre difficulté apparaît donc dans le choix des caractères à étudier : les effets d'une variation des fréquences de recombinaison ne seront nets que pour des caractères déterminés par des loci liés de façon assez étroite. Les taux de recombinaison entre loci de $0,5 \%$ à $30 \%$ rencontrés pour le taux moyen de $19,6 \%$ sont trop élevés. Cette nécessité de travailler sur des loci étroitement liés, pour mettre en évidence des différences liées aux taux de recombinaison, avait été soulignée par Latter (1965) dans le cas de 2 loci portant des gènes majeurs.

Enfin, pour interpréter les résultats, il est nécessaire de bien connaître le déterminisme génétique des caractères.

L'étude des conséquences de la recombinaison par des calculs ou des simulations à partir de modèles génétiques permet de s'affranchir de ces contraintes expérimentales mais la question de l'adéquation du modèle à la réalité biologique se pose alors. La caractérisation de segments chromosomiques à effet favorable ou défavorable impliqués dans le contrôle de caractères quantitatifs (QTL), le suivi de ces marqueurs dans des descendances, associé à une approche classique des caractères quantitatifs, permettront peut-être de mieux visualiser les conséquences de la recombinaison méiotique sur la variabilité génétique et son expression phénotypique, tout en prenant en compte l'organisation de l'information génétique (couplage ou répulsion) à l'aide de croisements appropriés. 


\section{La recombinaison dans la pratique de la sélection}

Du point de vue de l'amélioration des plantes, les résultats des simulations sans sélection suggèrent que, si le sélectionneur pouvait accroître la fréquence des recombinaisons afin de rompre les liaisons défavorables, les possibilités d'obtention et d'identification d'individus transgressifs n'en seraient pas augmentées pour autant à court terme. Notre simulation de sélection récurrente montre cependant qu'à plus long terme la recombinaison intrachromosomique accélère le progrès génétique, bien qu'elle ne conduise pas à des variances génétiques plus élevées au niveau des populations. Ce résultat va dans le sens des travaux de Hanson (1959) qui a démontré que plusieurs cycles d'intercroisements sont nécessaires pour obtenir la cassure et la réassociation des segments chromosomiques chez les autogames. L'améliorateur aura donc intérêt à choisir des méthodes où les recombinaisons intrachromosomiques efficaces, entre segments hétérozygotes, sont favorisées : approche plus lente de l'homozygotie par des croisements pleinsfrères chez les plantes allogames (Cornelius et Dudley, 1974), plans complexes d'intercroisements faisant intervenir plusieurs géniteurs, tant pour les plantes allogames qu'allogames (Empig, 1971; Doussinault et Trottet, 1981; Gallais, 1981). L'absence de différence constatée entre les populations correspondant aux taux moyens de $19,6 \%$ et $45 \%$ montre que de telles méthodes (ici plan de croisement à 3 étages) peuvent permettre de bien gérer la variabilité du matériel végétal de départ et d'obtenir un progrès satisfaisant.

Dans ce contexte, l'utilisation de gènes favorisant la recombinaison intra-chromosomique ne semble présenter d'intérêt que si les loci desquels dépend le progrès génétique sont très liés ou si les croisements sont difficiles à mettre en œuvre.

\section{RÉFÉRENCES}

Bulmer MG (1971) The effect of selection on genetic variability. Am Nat 105, 201-211
Bulmer MG (1974) Linkage disequelibrium and genetic variability. Genet Res 23, 281-283

Cornelius PL, Dudley JW (1974) Effect of inbreeding and full-mating in mating in maize population. Crop Sci $14,815-819$

Doussinault G, Trottet M (1981) Application de la sélection récurrente aux espèces autogames ou allogames non strictes : allogamisation des autogames. Sélect Fr 29, 25-33

Empig LT (1971) A program designed to maximize genetic variability in breeding of self-fertilizing crops. Philipp Agric 54, 319-324

Gallais A (1974) Covariances between arbitrary relatives with linkage and epistasis in the case of linkage disequilibrium. Biometrics 30, 429-446

Gallais A (1981) Amélioration des populations en vue de la création de variétés. Sélect Fr 29, 5-23

Hallauer AR, Miranda JB (1981) Quantitative genetics in maize breeding. lowa State University, Ames, $468 p$

Hanson WD (1959) The breakup of initial linkage blocks under selected mating systems. Genetics 44, 857-868

Latter BDH (1965) The reponse to artificial selection due to autosomal genes of large effect. I. Changes in frequency at an additive locus. Aust $J$ Biol Sci $18,585-598$

Mather K (1941) Variation and selection of polygenic characters. J Genet 41, 159-193

Mather K (1942) The balance of polygenic combinations. J Genet 43, 309-336

Mather K, Jinks JL (1977) Introduction to biometrical genetics. Cornell Univ Press, Ithaca, New York

Miller PA, Rawling JO (1967) Break up of initial blocks through intermating in cotton breeding populations. Crop Sci 7, 199-204

Robert N, Kervella J, Fouilloux G (1993) Influence de la recombinaison sur la variabilité génétique. I. Étude expérimentale. agronomie 13, 275-281

Snape JW (1976) A theoretical comparison of diplojsed haploid and single seed descent population. Heredity 36, 275-277

Snape JW, Simpson E (1981) The genetical expectation of doubled haploid lines derived from different filial generations. Theor Appl Genet 60, 123-133

Spiess EB, Allen AC (1961) Release of genetic variability through recombinaison. VI. Second and third chromosomes of Drosophila melanogaster. Genetics 46, 1531-1553

Verrier E, Colleau JJ, Foulley JL (1990) Predicting cumulated response to directional selection in finite panmictic populations. Theor App/ Genet 79, 833-840 